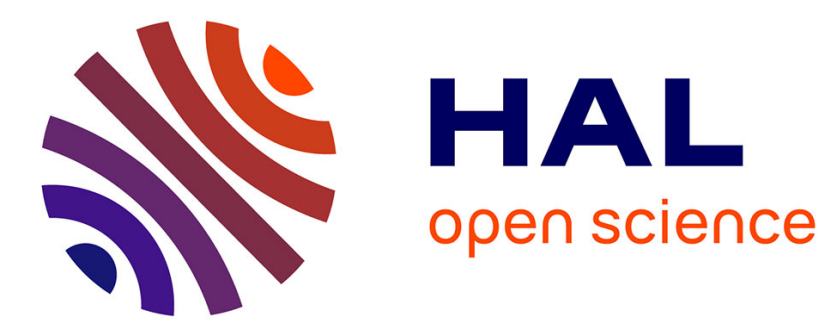

\title{
Symmetric Decentralized Interference Channels with Noisy Feedback
}

\author{
Samir M. Perlaza, Ravi Tandon, H. Vincent Poor
}

\section{To cite this version:}

Samir M. Perlaza, Ravi Tandon, H. Vincent Poor. Symmetric Decentralized Interference Channels with Noisy Feedback. IEEE Intl. Symposium on Information Theory (ISIT), Jun 2014, Honolulu, HI,

United States. hal-00983991

\section{HAL Id: hal-00983991 \\ https://hal.inria.fr/hal-00983991}

Submitted on 26 Apr 2014

HAL is a multi-disciplinary open access archive for the deposit and dissemination of scientific research documents, whether they are published or not. The documents may come from teaching and research institutions in France or abroad, or from public or private research centers.
L'archive ouverte pluridisciplinaire HAL, est destinée au dépôt et à la diffusion de documents scientifiques de niveau recherche, publiés ou non, émanant des établissements d'enseignement et de recherche français ou étrangers, des laboratoires publics ou privés. 


\title{
Symmetric Decentralized Interference Channels with Noisy Feedback
}

\author{
Samir M. Perlaza*‡, Ravi Tandon ${ }^{\dagger}$ and H. Vincent Poor $^{\ddagger}$ \\ * Institut National de Recherche en Informatique et Automatique (Inria), Villeurbanne, France \\ $\dagger$ Hume Center and Department of ECE, Virginia Tech, Blacksburg, VA \\ $\ddagger$ Department of Electrical Engineering. Princeton University, Princeton, NJ \\ samir.perlaza@inria.fr, tandonr@vt.edu,poor@princeton.edu
}

\begin{abstract}
In this paper, all the rate-pairs that are achievable at a Nash equilibrium (NE) in the two-user linear deterministic symmetric decentralized interference channel (LD-S-DIC) with noisy feedback are identified. More specifically, the Nash region (NR) of the LD-S-DIC with noisy feedback is fully characterized. The relevance of these rate-pairs is that once they are achieved by using NE transmit-receive configurations, none of the transmitter-receiver pairs can increase their individual rates by unilaterally changing their configurations. More importantly, it is shown that the NR of the LD-S-DIC with noisy feedback is larger than the NR of the LD-S-DIC without feedback only in certain cases. When interference is stronger than the desired signals, a larger NR is observed only if the signal to noise ratios (SNRs) of the feedback links are higher than the SNRs of the direct links. Conversely, when desired signals are stronger than interference, a larger NR is observed only if the SNRs of the feedback links are higher than both the signal to interference ratios (SIRs) and the interference to noise ratios (INRs) of the direct links. Previous results, namely the NE region of the two-user $L D-S-D I C$ without feedback and with perfect output feedback are obtained as special cases of the results presented in this contribution.
\end{abstract}

\section{INTRODUCTION}

The traditional role of feedback in wireless communications systems has been to improve their reliability. Recently, a transformative role of feedback has emerged in the context of interference networks: harnessing interference as side information [1], [2], [3]. More specifically, when a transmitter receives a feedback signal from its intended receiver, it obtains a degraded version of the sum of its own transmitted signal and the interfering signals from other transmitters. This implies that subject to a finite delay, both transmitters know at least partially the information transmitted by each other. This induces a type of cooperation between transmitters in the sense that they share their information bits. This effect is a consequence of the broadcast nature of wireless channels, and thus, even if this sort of cooperation is not explicitly desired by the transmitters, it incontestably appears as long as all code books are known by all transmitter-receiver pairs.

This paper studies the benefits of feedback in fully decentralized wireless networks, that is, networks in which transmitter-receiver pairs are solely interested in transmitting at their highest achievable rates, despite the rates that other transmitter-receiver pairs might achieve. To this end, the twouser Gaussian decentralized interference channel (DIC) with

This research was supported in part by the Air Force Office of Scientific Research under MURI Grant FA9550-09-1-0643 and in part by the Marie Curie Outgoing Fellowship Program under Award No. FP7-PEOPLE-IOF2011-298532. noisy feedback is studied using a linear deterministic (LD) approximation and tools from game theory. In this setting, it is shown that the sort of cooperation induced by feedback allows both links to achieve Nash equilibria (NEs) that lie in the Pareto optimal region, i.e., the set of sum-rate optimal transmitter-receiver configurations. All the rate-pairs that are achievable at an NE in the two-user linear deterministic symmetric decentralized interference channel (LD-S-DIC) with noisy feedback are identified. More importantly, it is shown that the set of rate pairs that are achievable at an NE, i.e., Nash region of the LD-S-DIC with noisy feedback, is larger than the Nash region of the LD-S-DIC without feedback only in certain cases. That is, the benefits of feedback appear only if certain conditions are met. When interference is stronger than the desired signals, a larger NR is observed only if the signal to noise ratios (SNRs) of the feedback links are higher than the SNRs of the direct links. Conversely, when desired signals are stronger than interference, a larger NR is observed only if the SNRs of the feedback links are higher than both the signal to interference ratios (SIRs) and the interference to noise ratios (INRs) of the direct links. Interestingly, the $\mathrm{NE}$ region of the LD-DIC without feedback [4] and the NE region of the LD-DIC with perfect output feedback [5], [6] are obtained as corollaries of the main theorem presented in this paper.

\section{LineAR DETERMinistic IC With NoISY FEEDBACK}

Consider the two-user Gaussian DIC with noisy feedback shown in Fig. 1. Transmitter $i$, with $i \in\{1,2\}$, communicates with receiver $i$ during $T$ consecutive blocks subject to the interference produced by transmitter $j \in\{1,2\} \backslash\{i\}$. The linear deterministic approximation [7] of this decentralized channel, known as the two-user LD-DIC with noisy feedback, can be described by six parameters: $\vec{n}_{11}, \vec{n}_{22}, n_{12}, n_{21}$, $\overleftarrow{n}_{11}$ and $\overleftarrow{n}_{22}$. The parameter $\vec{n}_{i i} \geqslant 0$ captures the signal strength from transmitter $i$ to receiver $i ; n_{i j} \geqslant 0$ captures the interference strength from transmitter $j$ to receiver $i$; and $\overleftarrow{n}_{i i}$ captures the feedback signal strength from receiver $i$ to transmitter $i$. Let $q=\max _{(i, j) \in\{1,2\}^{2}} \max \left(n_{i j}, \overleftarrow{n}_{i i}, \vec{n}_{i i}\right)$ be strictly positive and finite. Then, the input-output relation of the two user LD-DIC is

$$
\begin{aligned}
& \overrightarrow{\boldsymbol{y}}_{1}^{(t)}=\boldsymbol{S}^{q-\vec{n}_{11}} \boldsymbol{x}_{1}^{(t)}+\boldsymbol{S}^{q-n_{12}} \boldsymbol{x}_{2}^{(t)}, \text { and } \\
& \overrightarrow{\boldsymbol{y}}_{2}^{(t)}=\boldsymbol{S}^{q-n_{21}} \boldsymbol{x}_{1}^{(t)}+\boldsymbol{S}^{q-\vec{n}_{22}} \boldsymbol{x}_{2}^{(t)},
\end{aligned}
$$




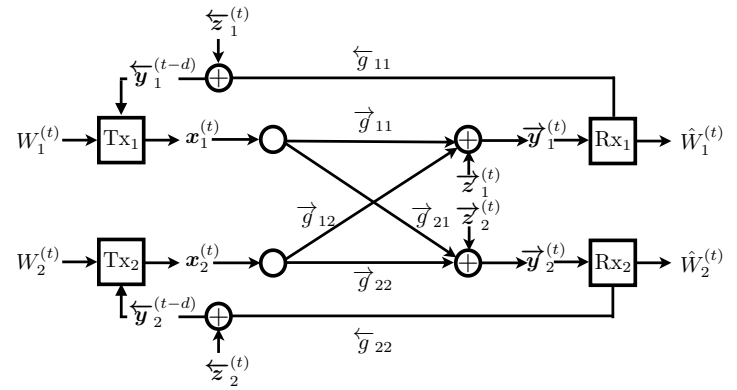

Fig. 1. Two-user decentralized Gaussian interference channel with (delayed) noisy feedback.

where $\boldsymbol{x}_{i}^{(t)}=\left(x_{i, 1}^{(t)}, \ldots, x_{i, q}^{(t)}\right)^{T}$ is the channel input vector generated by transmitter $i$ and $\boldsymbol{y}_{i}^{(t)}=\left(y_{i, 1}^{(t)}, \ldots, y_{i, q}^{(t)}\right)^{T}$ is the channel output received by receiver $i$ during block $t \in$ $\{1, \ldots, T\}$. The matrix $S$ is a $q \times q$ lower shift matrix and all additions and multiplications are over a binary field.

A noisy feedback link from receiver $i$ to transmitter $i$ allows at the end of each block $t$ the observation of a degraded version of the output $\overrightarrow{\boldsymbol{y}}_{i}^{(t-d)}$ at transmitter $i$, within a finite delay of $d$ blocks. Thus, the feedback signal available at transmitter $i$ at block $t$ is

$$
\overleftarrow{\boldsymbol{y}}_{i}^{(t)}=\boldsymbol{S}^{q-\overleftarrow{n}_{i i}} \overrightarrow{\boldsymbol{y}}_{i}^{(t-d)}
$$

Let $M_{i}$ be the number of information bits $b_{i, 1}^{(t)}, \ldots, b_{i, M_{i}}^{(t)}$ sent by transmitter $i$ at every block $t$. Hence, the encoder of transmitter $i$, during block $t>d$, can be modeled as a deterministic mapping $f_{i}^{(t)}:\left\{1, \ldots, 2^{M_{i}}\right\} \times\{0,1\}^{(t-d) \cdot q} \rightarrow\{0,1\}^{q}$ such that $\boldsymbol{x}_{i}^{(t)}=f_{i}^{(t)}\left(k, \overleftarrow{\boldsymbol{y}}_{i}^{(1)}, \ldots, \overleftarrow{\boldsymbol{y}}_{i}^{(t-d)}\right)$, where $k$ is the index of the message to be transmitted and $\overleftarrow{\boldsymbol{y}}_{i}^{(1)}, \ldots, \overleftarrow{\boldsymbol{y}}_{i}^{(t-d)}$ are all previous channel outputs available at transmitter $i$ at block $t$. Note that for blocks for which $t \leqslant d$, the encoder is a mapping $f_{i}^{(t)}:\left\{1, \ldots, 2^{M_{i}}\right\} \rightarrow\{0,1\}^{q}$ for which the symbols $\boldsymbol{x}_{i}^{(t)}=f_{i}^{(t)}(k)$ do not depend on the previous degraded channel outputs $\overleftarrow{\boldsymbol{y}}_{i}^{(1)}, \ldots, \overleftarrow{\boldsymbol{y}}_{i}^{(t-d)}$. At the end of the complete transmission, after block $T$, receiver $i$ uses the channel outputs $\overrightarrow{\boldsymbol{y}}_{i}^{(1)}, \ldots, \overrightarrow{\boldsymbol{y}}_{i}^{(T)}$ to generate estimates $\hat{b}_{i, \ell}^{(t)}$ of the transmitted bits $b_{i, \ell}^{(t)}, \forall(\ell, t) \in\left\{1, \ldots, M_{i}\right\} \times\{1, \ldots, T\}$. The average bit error probability of transmitter $i$ during a transmission of $T$ blocks, denoted by $p_{i}^{(T)}$, is calculated as follows:

$$
p_{i}^{(T)}=\frac{1}{T \cdot M_{i}} \sum_{t=1}^{T} \sum_{\ell=1}^{M_{i}} \mathbb{1}_{\left\{\hat{b}_{i, \ell}^{(t)} \neq b_{i, \ell}^{(t)}\right\}} .
$$

The rate pair $\left(R_{1}, R_{2}\right) \in \mathbb{R}_{+}^{2}$ is achievable, if there exists at least one pair of codebooks with the corresponding encoding functions $f_{1}$ and $f_{2}$ such that the average bit error probability (4) can be made arbitrarily small by letting the number of channel uses $T$ grow to infinity.

The aim of transmitter $i$ is to autonomously choose its transmit configuration $s_{i}$ in order to maximize its achievable rate $R_{i}$. More specifically, the transmit configuration $s_{i}$ can be described in terms of the number of information bits per block $M_{i}$, the codebook, the encoding functions $f_{i}$, etc. Note that the rate achieved by receiver $i$ depends on both configurations $s_{1}$ and $s_{2}$ due to the mutual interference naturally arising in wireless channels. This reveals the competitive interaction between both links in the LD-DIC and justifies the use of tools from game theory in its analysis.

\section{A. Symmetric Linear Deterministic Approximation}

A particular case of the LD-DIC model is the symmetric case in which $\vec{n}=\vec{n}_{11}=\vec{n}_{22}, m=n_{12}=n_{21}$ and $\overleftarrow{n}=$ $\overleftarrow{n}_{11}=\overleftarrow{n}_{22}$. The capacity region of the two-user symmetric LD-DIC with noisy feedback is denoted by $\mathcal{C}_{(\vec{n}, \overleftarrow{n}, m)}$ and it is fully characterized by Theorem 1 in [8].

Lemma 1 (Theorem 1 in [8]): The capacity region $\mathcal{C}_{(\vec{n}, \overleftarrow{n}, m)}$ of the two-user LD-DIC with noisy feedback corresponds to the set of non-negative rate pairs $\left(R_{1}, R_{2}\right)$ that satisfy

$$
\begin{gathered}
R_{i} \leqslant \max (\vec{n}, m), i \in\{1,2\} \\
R_{i} \leqslant \vec{n}+(\overleftarrow{n}-\vec{n})^{+}, i \in\{1,2\} \\
R_{1}+R_{2} \leqslant(\vec{n}-m)^{+}+\max (\vec{n}, m) \\
R_{1}+R_{2} \leqslant 2 \max \left((\vec{n}-m)^{+}, m\right) \\
+2 \min \left((\vec{n}-m)^{+},\left(\overleftarrow{n}-\max \left((\vec{n}-m)^{+}, m\right)\right)^{+}\right) \\
R_{1}+2 R_{2} \leqslant(\vec{n}-m)^{+}+\max (\vec{n}, m) \\
+\min \left((\vec{n}-m)^{+},\left(\overleftarrow{n}-\max \left((\vec{n}-m)^{+}, m\right)\right)^{+}\right) \\
+\max \left((\vec{n}-m)^{+}, m\right) \\
2 R_{1}+R_{2} \leqslant(\vec{n}-m)^{+}+\max (\vec{n}, m) \\
+\min \left((\vec{n}-m)^{+},\left(\overleftarrow{n}-\max \left((\vec{n}-m)^{+}, m\right)\right)^{+}\right) \\
+\max \left((\vec{n}-m)^{+}, m\right) .
\end{gathered}
$$

\section{B. Game Formulation}

The competitive interaction through mutual interference between the transmitters in the two-user DIC can be modeled by the following game in normal-form:

$$
\mathcal{G}=\left(\mathcal{K},\left\{\mathcal{A}_{k}\right\}_{k \in \mathcal{K}},\left\{u_{k}\right\}_{k \in \mathcal{K}}\right) .
$$

The set $\mathcal{K}=\{1,2\}$ is the set of players, that is, the set of transmitter-receiver pairs. The sets $\mathcal{A}_{1}$ and $\mathcal{A}_{2}$ are the sets of actions of players 1 and 2, respectively. An action of player $i$, which is denoted by $s_{i} \in \mathcal{A}_{i}$, is basically its transmit/receive configuration as described above. The utility function of player $i$ is $u_{i}: \mathcal{A}_{1} \times \mathcal{A}_{2} \rightarrow \mathbb{R}_{+}$and it is defined as the achieved rate of transmitter $i$, that is,

$$
u_{i}\left(s_{1}, s_{2}\right)= \begin{cases}R_{i}\left(s_{1}, s_{2}\right), & \text { if } \quad \forall t \in\{1, \ldots, T\}, p_{i}^{(T)}<\epsilon, \\ 0, & \text { otherwise }\end{cases}
$$

where $\epsilon>0$ is an arbitrarily small number and $R_{i}\left(s_{1}, s_{2}\right)$ denotes a transmission rate achievable with the configurations $s_{1}$ and $s_{2}$. Often, the rate $R_{i}\left(s_{1}, s_{2}\right)$ is written as $R_{i}$ for the sake of simplicity. However, every non-negative rate is associated with a particular pair of transmit configurations $s_{1}$ 
and $s_{2}$. It is worth noting that there might exist several transmit configurations that achieve the same rate pair $\left(R_{1}, R_{2}\right)$.

Some action profiles $s=\left(s_{1}, s_{2}\right) \in \mathcal{A}_{1} \times \mathcal{A}_{2}$ are particularly important in the analysis of this game. These actions profiles are referred to as $\eta$-Nash equilibria $(\eta$-NE) and obey the following definition:

Definition 1 ( $\eta$-Nash Equilibrium): In the game $\mathcal{G}=$ $\left(\mathcal{K},\left\{\mathcal{A}_{k}\right\}_{k \in \mathcal{K}},\left\{u_{k}\right\}_{k \in \mathcal{K}}\right)$, an action profile $\left(s_{1}^{*}, s_{2}^{*}\right)$ is an $\eta$ Nash equilibrium if $\forall i \in \mathcal{K}$ and $\forall s_{i} \in \mathcal{A}_{i}$,

$$
u_{i}\left(s_{i}, s_{j}^{*}\right) \leqslant u_{i}\left(s_{i}^{*}, s_{j}^{*}\right)+\eta .
$$

From Def. 1, it becomes clear that if $\left(s_{1}^{*}, s_{2}^{*}\right)$ is an $\eta$-Nash equilibrium, then none of the transmitters can increase its own transmission rate more than $\eta$ bits per block by changing its own transmit configuration and keeping the average bit error probability arbitrarily close to zero. Thus, at a given $\eta$-NE, every transmitter achieves a utility (transmission rate) that is $\eta$-close to its maximum achievable rate given the transmit configuration of the other transmitter. Note that if $\eta=0$, then the classical definition of NE is obtained [9]. The relevance of the notion of equilibrium is that at any NE, every transmitter configuration is optimal with respect to the configuration of the other transmitters. The following investigates the set of rate pairs that can be achieved at an NE. This set of rate pairs is known as the Nash region.

Definition 2 (Nash Region): An achievable rate pair $\left(R_{1}, R_{2}\right)$ is said to be in the Nash region of the game $\mathcal{G}=\left(\mathcal{K},\left\{\mathcal{A}_{k}\right\}_{k \in \mathcal{K}},\left\{u_{k}\right\}_{k \in \mathcal{K}}\right)$ if there exists an action profile $\left(s_{1}^{*}, s_{2}^{*}\right)$ that is an $\eta$-Nash equilibrium for an arbitrarily small $\eta$ and the following holds:

$$
u_{1}\left(s_{1}^{*}, s_{2}^{*}\right)=R_{1} \quad \text { and } \quad u_{2}\left(s_{1}^{*}, s_{2}^{*}\right)=R_{2} .
$$

The following section studies the NE region of the game $\mathcal{G}$ in (11).

\section{MAIN RESULT}

This section presents a complete characterization of the NE region (Def. 2) of the symmetric LD-DIC with noisy feedback with parameters $\vec{n}, \overleftarrow{n}$ and $m$. Let $\mathcal{N}_{(\vec{n}, \overleftarrow{n}, m)}$ denote such an $\mathrm{NE}$ region and consider the following region:

$$
\mathcal{B}_{(\vec{n}, \overleftarrow{n}, m)}=\left\{\left(R_{1}, R_{2}\right): L \leqslant R_{i} \leqslant U, \forall i \in\{1,2\}\right\}
$$

where $L$ and $U$ are defined as follows:

$$
\begin{gathered}
L=(\vec{n}-m)^{+} \text {and } \\
U=\left\{\begin{array}{lll}
U^{(a)} & \text { if } & m \geqslant \vec{n} \\
U^{(b)} & \text { if } & m \leqslant \vec{n}
\end{array}\right.
\end{gathered}
$$

with

$$
\begin{aligned}
U^{(a)}= & \min (\max (\vec{n}, \overleftarrow{n}), m), \text { and } \\
U^{(b)}= & \max (\vec{n}, m)-\min \left((\vec{n}-m)^{+}, m\right) \\
& +\left(\min \left((\vec{n}-m)^{+}, m\right)-(\max (\vec{n}, m)-\overleftarrow{n})\right)^{+}
\end{aligned}
$$

The following theorem fully characterizes the Nash region $\mathcal{N}_{(\vec{n}, \overleftarrow{n}, m)}$ in terms of the region $\mathcal{B}_{(\vec{n}, \overleftarrow{n}, m)}$ in (15) and the capacity region $\mathcal{C}_{(\vec{n}, \overleftarrow{n}, m)}$ described by Lemma 1
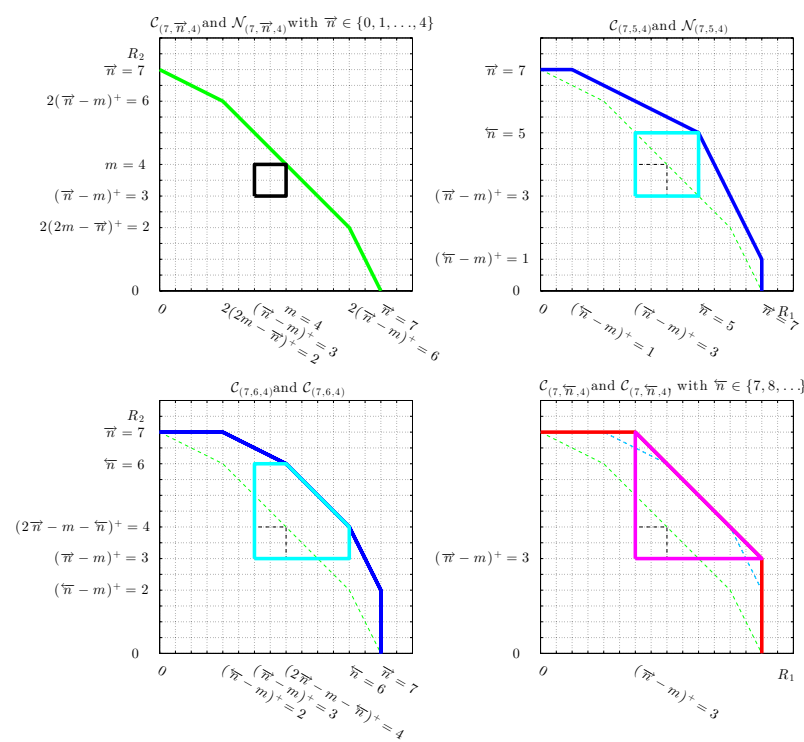

Fig. 2. Illustration of $\mathcal{C}_{(7,0,4)}$ (green line) and $\mathcal{N}_{(7,0,4)}$ (black line) in the left-top figure; $\mathcal{C}_{(7,5,4)}$ (blue line) and $\mathcal{N}_{(7,5,4)}$ (cyan line) in the right-top figure; $\mathcal{C}_{(7,6,4)}$ (blue line) and $\mathcal{N}_{(7,6,4)}$ (cyan line) in the left-bottom figure; and $\mathcal{C}_{(7,0,4)}$ (red line) and $\mathcal{N}_{(7,7,4)}$ (magenta line) in the right-bottom figure.

Theorem 1: The Nash region of the two-user symmetric LD-DIC with noisy feedback, with parameters $\vec{n}, m$ and $\overleftarrow{n}$, is

$$
\mathcal{N}_{(\vec{n}, \overleftarrow{n}, m)}=\mathcal{B}_{(\vec{n}, \overleftarrow{n}, m)} \cap \mathcal{C}_{(\vec{n}, \overleftarrow{n}, m)}
$$

The rest of this section presents examples and connections with existing results that provide some insight into the line followed by the proof of Theorem 1 .

\section{A. Examples of Nash Regions}

Consider the case of weak interference $\left(\frac{1}{2} \leqslant \alpha=\frac{m}{\vec{n}} \leqslant 1\right)$, for instance, $\vec{n}=7$ and $m=4$, with different levels of noise in the feedback channel, i.e., $\overleftarrow{n} \in\{0,1, \ldots\}$. In Fig. 2 the capacity region $\mathcal{C}_{(7, \overleftarrow{n}, 4)}$ and the Nash region $\mathcal{N}_{(7, \overleftarrow{n}, 4)}$ are plotted for each value of $n$.

The left-top plot in Fig. 2 shows the regions $\mathcal{C}_{(7, \overleftarrow{n}, 4)}$ (green line) and $\mathcal{N}_{(7, \overleftarrow{n}, 4)}$ (black line), with $\overleftarrow{n} \in\{0,1, \ldots, 4\}$. These regions correspond exactly to the capacity region and the $\mathrm{NE}$ region of the symmetric LD-DIC without feedback described in Theorem 1 in [10] and in Theorem 1 in [4], respectively. The following corollary formalizes this observation.

Corollary 1 (No Feedback): The Nash region of the symmetric LD-DIC without feedback $(\overleftarrow{n}=0)$, with parameters $\vec{n}$ and $m$, is $\mathcal{N}_{(\vec{n}, 0, m)}$.

A more interesting observation from Fig. 2 is that the capacity region and the Nash region remain the same for all $\overleftarrow{n} \in\{0,1, \ldots, 4\}$. Conversely, the right-top and left-bottom plots in Fig. 2 show capacity regions and NE regions that are larger than $\mathcal{C}_{(\vec{n}, 0, m)}$ and $\mathcal{N}_{(\vec{n}, 0, m)}$, respectively. Hence, from Theorem 1, two important conclusions can be drawn: $(a)$ when the desired signals are stronger than the interference, i.e., $\vec{n}>m$, for obtaining larger NE regions than the one obtained without feedback $\mathcal{N}_{(\vec{n}, 0, m)}$, i.e., to observe

$$
\left(\min \left((\vec{n}-m)^{+}, m\right)-(\max (\vec{n}, m)-\overleftarrow{n})\right)^{+}>0
$$


in (17), the following condition is necessary:

$$
\overleftarrow{n}>\max \left((\vec{n}-m)^{+}, m\right)
$$

This implies that for observing a noticeable effect on the Nash region due to the use of feedback, the SNRs $\overleftarrow{n}$ of the feedback links $\mathrm{Rx}_{i} \rightarrow \mathrm{Tx}_{i}$ must be superior to the SIRs $(\vec{n}-m)^{+}$and the INRs $m$ of the direct links $\mathrm{Tx}_{i} \rightarrow \mathrm{Rx}_{i}$. (b) When the interference is stronger than the desired signals, i.e., $m \geqslant$ $\vec{n}$, for observing a larger NE region than the one without feedback, the following condition is necessary:

$$
\overleftarrow{n}>\vec{n}
$$

in (17). That is, the feedback links must exhibit a higher SNRs $\overleftarrow{n}$ than the SNRs $\vec{n}$ of the direct links. Therefore, the sole existence of feedback links $\mathrm{Rx}_{i} \rightarrow \mathrm{Tx}_{i}$ is not a sufficient condition for enlarging the Nash region of the LD-S-DIC and some conditions need to be met. The following corollary formalizes this observation.

Corollary 2 (Noisy Feedback): Necessary conditions for observing $\mathcal{N}_{(\vec{n}, 0, m)} \subset \mathcal{N}_{(\vec{n}, \overleftarrow{n}, m)}$ with strict inclusion are

- $\overleftarrow{n}>\max \left((\vec{n}-m)^{+}, m\right)$ if $m<\vec{n}$; and

- $\overleftarrow{n}>\vec{n}$ if $m \geqslant \vec{n}$

The right-bottom plot in Fig. 2 shows the capacity $\mathcal{C}_{(7, \overleftarrow{n}, 4)}$ (red line) and $\mathrm{NE} \mathcal{N}_{(7, \overleftarrow{n}, 4)}$ (magenta line) regions, with $\overleftarrow{n} \in\{7,8, \ldots\}$. These plots correspond exactly to the capacity region of the LD-S-IC with perfect output feedback and the $\mathrm{NE}$ region of the LD-S-DIC with perfect output feedback described in [1] and [5], [6], respectively. More formally, note that in (17), the following inequality always holds for all $(\vec{n}, \overleftarrow{n}, m) \in \mathbb{N}^{3}$ :

$$
U \leqslant \max (\vec{n}, m),
$$

and strict equality only holds when the following condition is met:

$$
\overleftarrow{n} \geqslant \max (\vec{n}, m)
$$

This observation implies that, when the signal is stronger than the interference, i.e., $\vec{n} \geqslant m$, any improvement of the SNRs $\overleftarrow{n}$ of the feedback links beyond the SNRs $\vec{n}$ of the direct links does not enlarge the NE region. Similarly, when the interference is stronger than the desired signal, i.e., $m \geqslant \vec{n}$, improving the SNRs $\overleftarrow{n}$ of the feedback links beyond the INRs $m$ does not enlarge the NE region. The following corollary formalizes this observation.

Corollary 3 (Perfect-Output Feedback): The Nash region of the LD-S-DIC with perfect output feedback $(\overleftarrow{n} \geqslant$ $\max (\vec{n}, m))$ is $\mathcal{N}_{(\vec{n}, \max (\vec{n}, m)), m)}$.

From Cor. 3 and Cor. 2, the following inclusion holds:

$$
\mathcal{N}_{(\vec{n}, 0, m)} \subseteq \mathcal{N}_{(\vec{n}, \overleftarrow{n}, m)} \subseteq \mathcal{N}_{(\vec{n}, \max (\vec{n}, m), m)}
$$

In particular, from Cor. 2 and Cor. 3, it follows that strict inclusions hold in (24) when $\max \left((\vec{n}-m)^{+}, m\right)<\overleftarrow{n}<$ $\max (\vec{n}, m)$.

\section{B. Examples of Achievability}

Consider the case in which $\vec{n}=7, m=4$, and $\overleftarrow{n}=6$ (see the left-bottom plot in Fig. 2). In this case, the Nash region $\mathcal{N}_{(\vec{n}, \overleftarrow{n}, m)}$ is the convex hull of the rate pairs $(3,3)$ $(6,3),(6,4),(4,6)$ and $(3,6)$. The following shows the coding schemes that achieve an NE at each of these rate pairs.

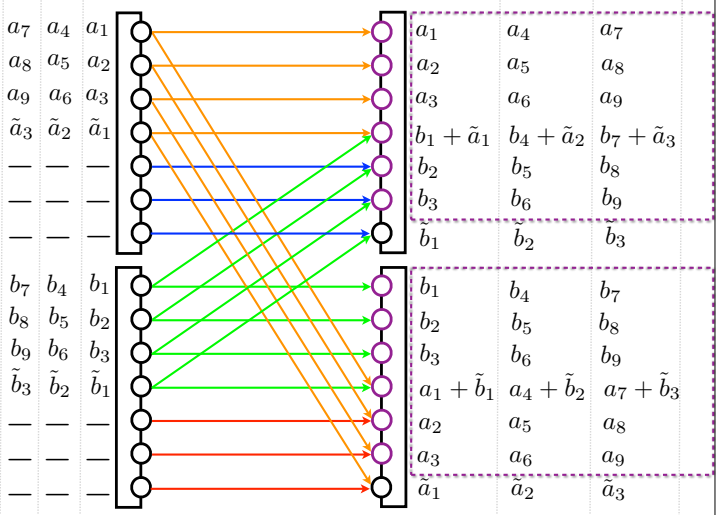

Fig. 3. Achievability scheme of the equilibrium rate pair $(3,3)$ of the NE region $\mathcal{N}_{(7,6,4)}$. Only the levels inside the purple dashed box are fed back to the corresponding transmitter. Note that $\tilde{a}_{1}, \tilde{a}_{2}, \ldots$ (resp. $\left.\tilde{b}_{1}, \tilde{b}_{2}, \ldots\right)$ are known at receiver 1 (resp. 2) and do not produce any interference at receiver 1 (resp. 2).

1) Achievability of the $N E$ rate pair $(3,3)$ : The rate pair $(3,3)$ is achievable when both receivers treat their mutual interference as noise (see Fig. 3). That is, transmitter $i$ sends its own information bits by using the top $(\vec{n}-m)^{+}$levels of the codeword $\boldsymbol{X}_{i}^{(t)}$ during the block $t$, which are received interference-free at receiver $i$. Note that transmitter 1 (resp. transmitter 2) also sends randomly generated symbols, denoted by $\tilde{a}_{1}, \tilde{a}_{2}, \ldots$ (resp. $\left.\tilde{b}_{1}, \tilde{b}_{2}, \ldots\right)$ in Fig. 3 . These symbols are assumed to be known at both transmitter 1 and receiver 1 (resp. transmitter 2 and receiver 2) and thus, they do not carry any information for link 1 (resp. link 2), however, they produce interference at receiver 2 (resp. receiver 1$)$. In this example, the sole objective of transmitting randomly generated bits is to prevent the other transmitter from sending new information bits and thus, increasing its transmission rate. As shown in Fig. 3, any attempt of transmitter $i$ to increase its individual rate by transmitting information bits in the other $\max (\vec{n}, m)-(\vec{n}-m)^{+}=m$ bits bounds the probability of error (4) away from zero. This is due to the interference produced by transmitter $j$ that affects these levels at receiver $i$.

Finally, note that if the transmitter-receiver pair $i$ uses its feedback channel, it does not bring any side information to transmitter $i$ to improve the coding scheme. Thus, given that no player can increase its individual rate given the transmit/receive configuration of the other transmitter-receiver pair, the rate pair $(3,3)$ is achieved at an NE. However, note that the NE pair $(3,3)$ is the worst NE in terms of sum-rate, which implies that treating interference as noise is the worst choice from both individual and global points of view.

2) Achievability of the $N E$ rate pair $(6,3)$ and $(3,6)$ : The rate pair $(6,3)$ is achieved thanks to feedback when transmitter 2 uses $\ell=3$ out of its $\min \left((\vec{n}-m)^{+}, m\right)$ top levels of the codeword $\boldsymbol{X}_{2}^{(t)}$ to transmit bits that have been previously transmitted by transmitter 1 and have produced interference at receiver 2 (see Fig. 4). When re-transmitted by transmitter 2 , these bits are used by receiver 2 to cancel the interference they have previously produced when they were transmitted by transmitter 1 ; and at receiver 1 , they do not produce any 


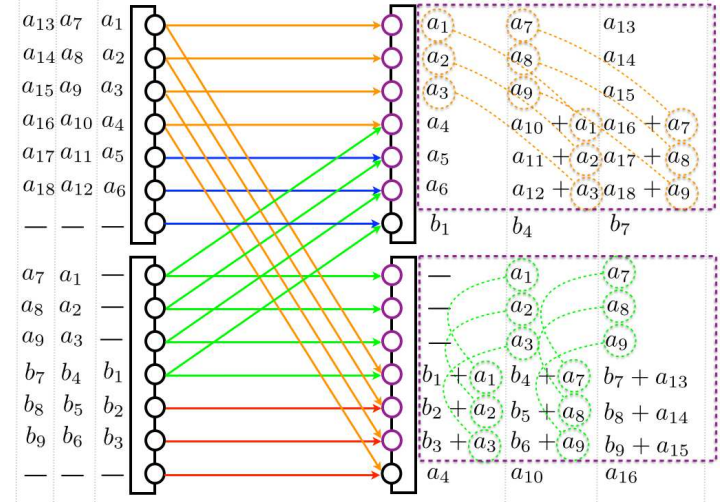

Fig. 4. Achievability scheme of the equilibrium rate pair $(6,3)$ of the NE region $\mathcal{N}_{(7,6,4)}$. Only the levels inside the purple dashed box are fed back to the corresponding transmitter.

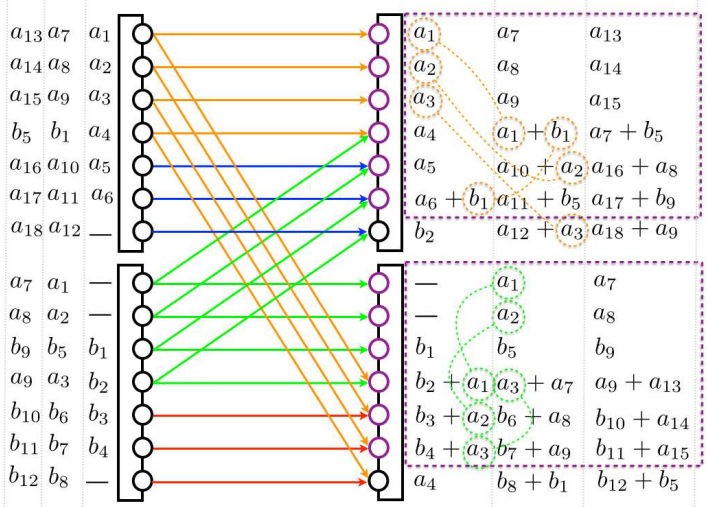

Fig. 5. Achievability scheme of the equilibrium rate pair $(6,4)$ of the NE region $\mathcal{N}_{(7,6,4)}$. Only the levels inside the purple dashed box are fed back to the corresponding transmitter.

interference since they have been previously received without any interference. Thus, in this example, transmitter 1 can transmit $\ell$ additional bits with respect to those it would be able to transmit if transmitter 2 does not use feedback to cancel interference.

In general, when transmitter $i$ uses $\ell$ of the top $\min \left((\vec{n}-m)^{+}, m\right)$ levels of its codeword $\boldsymbol{X}_{i}^{(t)}$ to retransmit bits that have been previously transmitted by transmitter $j$, it grants $\ell$ additional bits per block to transmitter $j$ with respect to the case in which those $\ell$ bits are used to send information bits corresponding to transmitter $i$. According to this reasoning, the following inequality holds: $\ell \leqslant \min \left((\vec{n}-m)^{+}, m\right)$.

3) Achievability of the NE rate pair $(6,4)$ and $(4,6)$ : The rate pair $(6,4)$ is achieved when both transmitter 1 and transmitter 2 use feedback to clean interference received in previous blocks (see Fig. 5). In this case, transmitter 1 uses $\ell_{1}=1$ levels out of the $\min \left((\vec{n}-m)^{+}, m\right)$ top levels of its codewords $\boldsymbol{X}_{1}^{(t)}$ to re-transmit $\ell_{1}$ bits previously transmitted by transmitter 2 in order to cancel its interference. This allows transmitter 2 to transmit an extra bit during each block with respect to the case in which transmitter 1 does not use feedback. This justifies that $R_{2}=4$. Transmitter 2 uses $\ell_{2}=3$ levels out of the $\min \left((\vec{n}-m)^{+}, m\right)$ top levels of its codewords $\boldsymbol{X}_{2}^{(t)}$ to re-transmit $\ell_{2}$ bits previously transmitted by transmitter 2 in order to cancel its interference. This justifies that $R_{1}=6$. Finally, note that any attempt of player $i$ to send new information bits at every block would bound its probability of error away from zero. Thus, none of the players can improve its transmission rate by changing its actual transmit-receive configuration. Hence, the rate pairs $(6,4)$ and $(4,6)$ are achievable at an NE.

\section{CONCLUSION}

This paper has presented a full characterization of the Nash region of the LD-DIC with noisy feedback. Previous results such as the Nash region of the LD-DIC without feedback and with perfect output feedback are obtained as particular cases of the results presented here. In particular, it has been shown that the existence of a feedback channel $\mathrm{Rx}_{i} \rightarrow \mathrm{Tx}_{i}$ in the decentralized interference channel is not a sufficient condition for enlarging its Nash region. Indeed, the feedback channels must satisfy some particular conditions for effectively enlarging the Nash region with respect to the case of the LD-SDIC without feedback. When the desired signals are stronger than the interference, a larger Nash region is observed only if the SNRs of the feedback links are superior to the SIRs and the INRs of the direct links. Conversely, when the interference is stronger than the desired signals, a larger Nash region is observed if the SNRs of the feedback links are superior to the SNRs of the direct links. That is, the Nash region of an LD-S-DIC with very noisy feedback links is identical to the Nash region of an LD-S-DIC without feedback.

\section{REFERENCES}

[1] C. Suh and D. N. C. Tse, "Feedback capacity of the Gaussian interference channel to within 2 bits," IEEE Transactions on Information Theory, vol. 57, no. 5, pp. 2667-2685, May 2011.

[2] A. Vahid, C. Suh, and A. S. Avestimehr, "Interference channels with ratelimited feedback," IEEE Transactions on Information Theory, vol. 58 , no. 5, pp. 2788-2812, May 2012.

[3] R. Tandon, S. Mohajer, and H. V. Poor, "On the symmetric feedback capacity of the K-user cyclic Z-interference channel," IEEE Transactions on Information Theory, vol. 59, no. 5, pp. 2713-2734, May 2013.

[4] R. A. Berry and D. N. C. Tse, "Shannon meets Nash on the interference channel," IEEE Transactions on Information Theory, vol. 57, no. 5, pp. 2821-2836, May 2011.

[5] S. M. Perlaza, R. Tandon, H. V. Poor, and Z. Han, "The Nash equilibrium region of the linear deterministic interference channel with feedback," in Proc. 50th Annual Allerton Conference on Communications, Control, and Computing, Monticello, IL, Oct. 2012.

[6] —, "Perfect output feedback in the two-user decentralized interference channel," (Submitted to) IEEE Transactions on Information Theory., Jun. 2013. http://arxiv.org/abs/1306.2878.

[7] S. Avestimehr, S. Diggavi, and D. N. C. Tse, "Wireless network information flow: A deterministic approach," IEEE Transactions on Information Theory, vol. 57, no. 4, pp. 1872-1905, Apr. 2011.

[8] S.-Q. Le, R. Tandon, M. Motani, and H. V. Poor, "Approximate capacity region for the symmetric Gaussian interference channel with noisy feedback," (Submitted to) IEEE Transactions on Information Theory, Dec. 2012. http://www.ece.vt.edu/tandonr/IC-Partial-Feedback.pdf.

[9] J. F. Nash, "Equilibrium points in n-person games," Proc. National Academy of Sciences of the United States of America, vol. 36, no. 1, pp. 48-49, Jan. 1950

[10] A. El Gamal and M. Costa, "The capacity region of a class of deterministic interference channels," IEEE Transactions on Information Theory, vol. 28, no. 2, pp. 343-346, Mar. 1982. 